\title{
Reliable Measures of Sarcopenia in Cirrhosis. Comment on: "The Relationship of Obesity, Nutritional Status and Muscle Wasting in Patients Assessed for Liver Transplantation, Nutrients 2019, 11, 2097"
}

\author{
Maryam Ebadi, Carlos Moctezuma-Velazquez ${ }^{\mathbb{D}}$, Rahima A. Bhanji and Aldo J. Montano-Loza * \\ Division of Gastroenterology \& Liver Unit, University of Alberta Hospital, Edmonton, AB T6G 2X8, Canada; \\ ebadi@ualberta.ca (M.E.); moctezum@ualberta.ca (C.M.-V.); rbhanji@ualberta.ca (R.A.B.) \\ * Correspondence: montanol@ualberta.ca; Tel.: +1-(780)-248-1892; Fax: +1-(780)-248-1895
}

Received: 25 November 2019; Accepted: 3 February 2020; Published: 24 March 2020

We read with interest the manuscript by Vidot et al. [1], investigating the relationship between nutritional status and muscle wasting (sarcopenia) in cirrhosis. In this study, the liver-specific subjective global assessment (SGA), which includes assessment of muscle wasting and subcutaneous fat loss [2], is shown to be insensitive for detecting muscle wasting in patients evaluated for liver transplantation (LT) [1], thus highlighting the need for reliable tools for the detection of muscle wasting and subcutaneous fat loss in clinical practice.

Although the present study contributes to evolving knowledge of sarcopenia and its complications in cirrhosis, some notes of caution are in order. Muscle wasting has been defined using sex-specific cut-offs for psoas muscle in patients undergoing elective resection for colorectal carcinoma [3]. The applicability of these sex-specific psoas muscle cut-offs is debatable in cirrhosis and may explain the high rate of sarcopenia; $97 \%$ of the male patients, constituting $70 \%$ of the study population, were identified as sarcopenic.

Psoas muscle measurement is rapid and detects sarcopenia better than individual anthropometric measurements; however, it has an asymmetrical shape, and the total area of both right and left psoas muscles accounts for approximately $10 \%$ to $13 \%$ [4,5] of the total muscle area at the third lumbar vertebra (L3) level. Of note, psoas muscle has limited capacity to recognize sarcopenic patients with higher waitlist mortality risk in cirrhosis [4]. Applying the validated definition of sarcopenia in North American patients with cirrhosis, which looks at the total skeletal muscle index (SMI) measured at L3 [6], we have previously demonstrated weak concordance between sarcopenia and malnutrition, as determined by SGA. We also found sarcopenia and not SGA predicts adverse clinical outcomes in cirrhosis. The concordance between malnutrition and sarcopenia was even weaker in overweight/obese patients [7]. Using SMI cut-offs, we found sarcopenia in 38\% of patients with cirrhosis (46\% in males, $25 \%$ in females).

The authors investigated potential predictors of sarcopenia and reported lower levels of testosterone in male and female patients with muscle wasting in comparison to their counterparts with normal muscle mass. The univariate analysis, however showed, every $1 \mathrm{nmol} / \mathrm{L}$ increase in testosterone level to be associated with a $20 \%$ increased risk of sarcopenia on the LT waiting list. This paradoxical association may be related to misclassification of patients using inappropriate modalities and cut-points. Lower testosterone levels were detected in sarcopenic male patients with cirrhosis [8], and treatment with testosterone was shown to improve muscle mass [9]. Lastly, lack of association between sarcopenia and wait list mortality might in part be due to misclassification of sarcopenia. Regardless of these 
limitations, the results of this study emphasize the importance of appropriate body composition assessment concurrent with nutritional assessments as part of the LT evaluation program.

Author Contributions: Search and selection of abstracts, review of the full-length articles and writing the final version of the article were performed by M.E., C.M.-V., R.A.B., and A.J.M.-L. All authors read and approved the final manuscript.

Funding: Maryam Ebadi has been awarded the Canadian Institutes of Health Research (CIHR)-Institute of Nutrition, Metabolism and Diabetes (INMD) Fellowship-Hepatology, in partnership with the Canadian Association for the Study of the Liver (CASL) and the Canadian Liver Foundation (CLF) (HGY-164788). Aldo J. Montano-Loza and Maryam Ebadi have been awarded the Canadian National Transplant Research Program (CNTRP), ATIF Innovation Grant Award 2018 and Canadian Liver Foundation (CLF), Operating Grant 2019.

Conflicts of Interest: The authors declare no conflict of interest.

\section{References}

1. Vidot, H.; Kline, K.; Cheng, R.; Finegan, L.; Lin, A.; Kempler, E.; Strasser, S.I.; Bowen, D.G.; McCaughan, G.W.; Carey, S.; et al. The Relationship of Obesity, Nutritional Status and Muscle Wasting in Patients Assessed for Liver Transplantation. Nutrients 2019, 11, 2097. [CrossRef] [PubMed]

2. Hasse, J.; Strong, S.; Gorman, M.A.; Liepa, G. Subjective global assessment: Alternative nutrition-assessment technique for liver-transplant candidates. Nutrition 1993, 9, 339-343. [PubMed]

3. Jones, K.I.; Doleman, B.; Scott, S.; Lund, J.N.; Williams, J.P. Simple psoas cross-sectional area measurement is a quick and easy method to assess sarcopenia and predicts major surgical complications. Colorectal Dis. 2015, 17, O20-O26. [CrossRef] [PubMed]

4. Ebadi, M.; Wang, C.W.; Lai, J.C.; Dasarathy, S.; Kappus, M.R.; Dunn, M.A.; Carey, E.J.; Montano-Loza, A.J.; From the Fitness, Life Enhancement, and Exercise in Liver Transplantation (FLEXIT) Consortium. Poor performance of psoas muscle index for identification of patients with higher waitlist mortality risk in cirrhosis. J. Cachexia Sarcopenia Muscle 2018, 9, 1053-1062. [CrossRef] [PubMed]

5. Rutten, I.J.; Ubachs, J.; Kruitwagen, R.F.; Beets-Tan, R.G.; Olde Damink, S.W.; Van Gorp, T. Psoas muscle area is not representative of total skeletal muscle area in the assessment of sarcopenia in ovarian cancer. J. Cachexia Sarcopenia Muscle 2017, 8, 630-638. [CrossRef] [PubMed]

6. Carey, E.J.; Lai, J.C.; Wang, C.W.; Dasarathy, S.; Lobach, I.; Montano-Loza, A.J.; Fitness, Life Enhancement, and Exercise in Liver Transplantation Consortium. A multicenter study to define sarcopenia in patients with end-stage liver disease. Liver Transplant. 2017, 23, 625-633. [CrossRef] [PubMed]

7. Moctezuma-Velazquez, C.; Ebadi, M.; Bhanji, R.A.; Stirnimann, G.; Tandon, P.; Montano-Loza, A.J. Limited performance of subjective global assessment compared to computed tomography-determined sarcopenia in predicting adverse clinical outcomes in patients with cirrhosis. Clin. Nutr. 2019, 38, 2696-2703. [CrossRef] [PubMed]

8. Moctezuma-Velázquez, C.; Low, G.; Mourtzakis, M.; Ma, M.; Burak, K.W.; Tandon, P.; Montano-Loza, A.J. Association between Low Testosterone Levels and Sarcopenia in Cirrhosis: A Cross-sectional Study. Ann. Hepatol. 2018, 17, 615-623. [CrossRef] [PubMed]

9. Sinclair, M.; Grossmann, M.; Hoermann, R.; Angus, P.W.; Gow, P.J. Testosterone therapy increases muscle mass in men with cirrhosis and low testosterone: A randomised controlled trial. J. Hepatol. 2016, 65, 906-913. [CrossRef] [PubMed]

(C) 2020 by the authors. Licensee MDPI, Basel, Switzerland. This article is an open access article distributed under the terms and conditions of the Creative Commons Attribution (CC BY) license (http://creativecommons.org/licenses/by/4.0/). 